\title{
Normalization of Heterographs in Chinese Northeast Dialect*
}

\author{
Ya-shu JIN \\ Yanbian University, Yanji, China
}

\begin{abstract}
The essence of heterographs is that there are various forms for one word. The form and the meaning are the two important aspects to normalize heterographs in Northeast dialect, while lexicology and philology are the two important aspects to study its subject nature. In term of lexicology, the meaning of words is the most important part in the arrangement and it determines the forming of heterographs. Therefore, the principle "in popular and easy-to-understand" is the most important principle in the normalization of heterographs. Meanwhile, Northeast dialect was the integration of multiple dialects and multiple ethnic languages, so that it should be easy-to-understand and making overall plans and take all factors into consideration, based on the clear expression. Northeast dialect has a rich cultural background and it is the most important carrier of the regional culture in Northeast. It reflects the cultural characteristics of northeast China, displaying the rich diversity of northeast culture. In fact, Northeast dialect itself is a kind of culture, recording the historical development of Northeast language. Normalization of heterographs in Northeast dialect is good in realistic instructive significance for developing and protecting Northeast dialect.
\end{abstract}

Keywords: heterographs, Northeast dialect, normalization methods and principles

\section{Introduction}

Northeast dialect has a rich cultural background and it is the most important carrier of the regional culture in Chinese Northeast, embodying the local feature and regional cultures from different regions. Northeast of China, in a narrow sense, refers to the three northeast provinces of China today (Liaoning province, Jilin province, and Heilongjiang province). In the broad sense, it includes the three northeast provinces of China and the five league cities belonging to Inner Mongolia at ancient time (Hulunbeier City, Tongliao, Chifeng, Hinggan League, and Xilin Gol League).

As a kind of subdialect of basic dialect, Northeast dialect is different from Mandarin, even if they are very similar with each other. It has experienced a complex languages and nations merging process when Northeast dialect came into being. In the process, a large number of heterographs have emerged. It has a positive influence for promoting Mandarin and dialect development to make a normalization of the heterographs in Northeast dialect as far as possible.

It has been nearly a half century since Mr. YIN Huan-xian wrote the article "Discussion on the Criterion of Words' Written Forms" in 1962, and then it was published in the famous publication "Chinese". So far, the

\footnotetext{
${ }^{*}$ Acknowledgements: Thanks for the fund supported by Jilin Provincial Education Department (No.2015.17 The 12th Five-Year of Social Science Research).

Ya-shu JIN, associate professor, M.A., College of Humanities and Social Science, Yanbian University, Yanji, China.
} 
academic circle has been always study about heterographs. But most of scholars almost have the same opinion that the essence of heterographs is that there are various forms for one word.

\section{The Subject Nature and Significance of the Normalization of Heterographs in Northeast Dialect}

\section{In Term of Lexicology and Philology}

The reason why the subject nature is in discussion is that it determines the direction of study. Different acknowledges of subject nature lead to different guiding ideologies and practical ideas even solving principles. The correct understanding of subject nature influences the outcome of study to a great extent.

For the nature of normalization of heterographs, there is a kind of view referring that

Normalization of heterographs refers to the issue of the same word in different writing styles. In other words, it means which character should be used. Basically, it can be classified into the one category with variant Chinese character, belonging to the criterion of Chinese characters. (GAO, 1993, p. 7)

"Normalization of heterographs is in close contact with lexicology, but it should be classified into the Chinese lexicology, not in lexicology, because what it focuses is on the criterion of written form of Characters" (GAO, 1993, p. 6).

Another idea is that the normalization of heterographs and variant Chinese character both focus on the writing of characters. However, the study of writing on characters should be based on the study of lexical system and semantic system, instead of only focusing on the writing form. Studies of meanings based on lexical studies and studies of form based on semantic studies both should be included in lexicology (LI, 2001, p. 54).

In another view,

There are similarities between normalization of heterographs and criterion of variant Chinese character, but there are also some differences. If a Chinese character has its variant, after normalization, the original heterographs will disappear without the variant which has been abandoned. (FU, 1985, p. 20)

In FU's opinion, "However, in variants word in modern Chinese, there is another situation. In each group of variants words, there is no variant relation among those words with the function to distinguish morphologies, instead, they have their own sound and meaning" (FU, 1985, p. 20).

As a matter of fact, the normalization of this kind of variants words equals to set the common meaning of several characters into one character, which means the redistribution of separation of meanings in Chinese characters. In term of this point, the criterion of variants words is fundamentally different from the criterion of heterographs. A considerable portion of the criterion of variants words belongs to the characters normalization, and a few of them are uninvolved in characters normalization. (FU, 1985, p. 21)

This kind of view is more comprehensive.

Concluding the previous researches, this paper holds the view that heterographs in Northeast dialect is a kind of written form with the same meaning and different forms, including homophones, dialectal heterographs, and Yixu Heterographs, which are in the same word element but different order in it. Heterographs in Northeast dialect involves two important issues: Vocabulary and Character. 


\section{It Has a Positive Significance for the Comprehensive Study of Northeast Dialect}

After studying the features and forming process of Northeast dialect, we found that it has an extensive connection with Mandarin. Northeast dialect is a unique linguistic culture phenomenon, with unique value in use and culture. In recent years, Northeast dialect has an extensive influence in the whole country, which diaplays the unique language artistic charming of it. Northeast dialect is not only a kind of communication tool in certain regions, but also an important carrier of northeast culture and the important part of Chinese culture. The diversity of dialect displays the diversity and the precious resources of Han culture.

Although Northeast dialect has attracted more and more attentions from scholars, the studies are still focus on phonetic, vocabulary, and rhetoric features. The study of form is still in virgin state. However, in all respects, on the study of Northeast dialect, the corpus of dialects is the very important support. From investigation, we found that it is always arbitrary in the selection of corpus. There is no doubt that it will influence the study and construction of Northeast dialect. The normalization and study of heterographs in Northeast dialect have some functions as following:

(1) Promote the development of native literature and expand the circulation of Northeast dialect. Northeast dialect is a kind of humorous, straightforward, and clear language which is full of imagination and most similar with Mandarin. The normalization of heterographs in dialect is helpful for native literature to choose practical vocabulary and for literature to be more pupolar;

(2) Provide absorbable materials to enrich Mandarin. Northeast dialect is a basic dialect in Han ethnic. The transition of Northeast dialect also occurred in Mandarin. Because of some special using environment, the vocabulary produced or used in Northeast dialect will become one of useful synonyms in Mandarin. The normalization of heterographs in dialect is necessary and significant for Mandarin to absorb some useful materials;

(3) Lay the foundation for the comprehensive study of the heterographs in Northeast dialect. The investigation of heterographs in Northeast dialect is helpful to lay the foundation for the comprehensive study of the heterographs in Northeast dialect. Besides, it will have a certain normative effect in the transition from dialect to written word and provides credible corpus for the usage and study of Northeast dialect.

\section{The Methods and Principles of Normalization of Heterographs in Northeast Dialect}

\section{Alternative Method}

The method of sorting out the heterographs is closely related to the determination of the its scope. When the principles of normalization is in discussion, the alternativeness of the congruent heterograph and subsumed heterograph is involved. In Gao Geng-sheng's view, heterographs includes congruent heterograph, subsumed heterograph, and crossed heterograph. On the sorting of congruent heterograph, the alternative method is used in total. On crossed heterograph, differential method is adopted. On the subsumed heterograph, both of the two methods are used. What we study in Northeast dialect is mainly congruent heterograph, so the alternative method should be used.

\section{The Principle on Sorting out of Heterographs in Northeast Dialect}

The principle on sorting out of heterographs in Mandarin is evolved from the principle on sorting out of heterographs. However, the sorting out of heterographs is more wide-ranging and more complex. HOU min 
(1992) and GAO Geng-sheng (1991) concluded the principles as four, including "being traditional, being simple, being clear, being accuracy". Agreeing on "Being traditional and being simple", LIU Jian-san (1996) added another three principles, "strictly control the number of words", "pay full attention to the visual image of Chinese characters", and "consider and weigh on the whole". TIAN Yu-ze (1993) added five principles, including "Based on current days", "sequencing", "pay attention to the emotional meaning", "making overall plans and take all factors into consideration", and "seize the moment". These principles above all emphasize on the guiding ideology of the sorting. It has certain reference value for the sorting of heterographs in Northeast dialect.

Because there is something special on the sorting of heterographs in Northeast dialect and there is not enough justification to inspect dialect words, the selection of words is only determined by using environment.

The differences in collection of corpus by different collectors lead that there is no justification for dictionary editing and articles writing. In this situation, linguistic sense is reliable. Comparing sever Northeast dialect dictionaries, we found that the heterographs phenomenon is obvious. Some heterographs focus on pronunciation, some focus on meaning, and some focus on the combination of them. We selected one of these dictionaries to sort out the heterographs in Northeast dialect.

We proposed the principle — being popular and clear, easy-to-understand, and making overall plans and take all factors into consideration, in other words, it means "Popularization, simple form, clear meaning, comprehensive consideration and easy understanding".

Being popular and clear. "Being popular and clear" means selecting words which are frequently used by most people and which have clear meanings. In terms of Mandarin, a group of heterographs could be quantitatively analyzed in computer, based on people's habits to apply the language. If there are marked differences on the frequency, they can be selected by the frequency ratio. However, the analysis in computer databases almost cannot be used in dialects. There is a long way to go for the establishment of database of Northeast dialect.

Chinese is a unity of form, sound, and meaning. For most of Chinese characters, there are morpheme records. Word's meaning is related to morpheme meaning and restricts the selection and combination of morphemes. On the contrary, morpheme meaning is good for understanding word's meaning. Therefore, clear meaning is not an important principle to normalize the word's form. Most of morphemes (Chinese characters) of heterographs have expressed clear meanings, so this principle plays a small role in selection of words. However, between two forms, if one is clear, while the other is vogue, this principle is important.

Easy-to-understand. "Easy-to-understand" focuses on the heterographs formed by commonly used characters and rarely-used characters. In general, the forms with rarely-used characters are always abandoned and the forms with commonly used characters are conserved. The form whose pronunciation is similar with the original will be used. In addition, when setting out the standard form, to avoid the mix on the pronunciation, characters with multi-sounds will not be selected. And the simple stroking is the better choice for less writing.

Making overall plans and take all factors into consideration. There are a lot of dialect words, onomatopoeia, and loanwords in Northeast dialect. Users often give them new meanings on different using environment, so that one word would have several different forms. Heterographs is common in the language which has writing form. Therefore, the principle proposed above all could be combined with each other, taking "Being popular and clear" as the main principle, taking "Easy-to-understand" as the second. 


\section{Conclusion}

Heterographs in Northeast dialect caused confusion to the written form communication, increasing difficulties of learning and using to people, adding barriers to information dealing of language and characters. The normalization of heterographs in Northeast dialect does not means the normalization of heterographs, neither of the simplification of traditional characters. In the process to come into being, it developed with the integration of cultures from multiple ethnics and multiple languages. The relationship between heterographs in Northeast dialect and language is complicated.

We classify the nature of normalization of heterographs in Northeast dialect into the category of lexicology and philology, proposing the principles of normalization, including being popular and clear, easy-to-understand, and making overall plans and take all factors into consideration. Because of the complicated rational investigation on heterographs in Northeast dialect, if the two principles, "being popular and clear" and "easy-to-understand", are contradictory, we should make overall plans and take all factors into consideration when establishing the standard of word form and try to make the normalization reasonable.

\section{References}

FU, Y. H. (1985). Questions about the normalization of heterographs. The Reforms of Chinese Characters, 1, 21-23.

GAO, J. S. (1991). About the normalization of heterographs. Language Planning, 1, 65-69.

GAO, J. S. (1993). About the normalization of heterographs again. Language Planning, 6, 7-11.

HOU, M. (1992). Questions of the normalization of heterographs. Language Planning, 3, 19-22.

LI, L. J. (2001). The core position in the normalization of heterographs. Lexicographical Studies, 7, 54-62.

LIU, J. S. (1996). The principles of normalization about the heterographs. Language Planning, 4, 8-9.

TIAN, Z. Y. (1993). Questions about the normalization of heterographs in morden Chinese. Journal of Jinzhou Teachers College, 1, $25-32$. 